UMD-PP-00-019

\title{
Breaking Parity Symmetry Using Extra Dimensions
}

\author{
R. N. Mohapatra ${ }^{1 *}$ and A. Pérez-Lorenzana ${ }^{1,2 \dagger}$ \\ 1 Department of Physics, University of Maryland, College Park, MD, 20742, USA \\ ${ }^{2}$ Departamento de Física, Centro de Investigación y de Estudios Avanzados del I.P.N. \\ Apdo. Post. 14-740, 07000, México, D.F., México.
}

(September, 1999)

\begin{abstract}
We present a new way to break parity symmetry in left-right symmetric models using boundary conditions on the fields residing in the fifth dimension. We also discuss the connection between the limits on the size of extra dimensions and the scale of right handed symmetry breaking obtained from the analysis of neutrinoless double beta decay in the case where the righthanded gauge symmetry is in the bulk..
\end{abstract}

pacs: 12.60.Cn; 11.30.Qc; 11.30.Er; 11.10.Kk

\section{INTRODUCTION}

The possible existence of hidden extra dimensions, motivated by superstring theories has provided a new way to look at many particle physics phenomena such as unification of couplings, baryon and lepton nonconservation [1] [1 and cosmological ones such as inflation, baryogenesis [8]. Perhaps one of the most interesting experimental fallouts of this line of research has come from the realization that extra dimensions almost as large as a millimeter could apparently be hidden from many extremely precise measurements that exist in particle physics leading to new searches for tests of gravitation at submillimeter distances. Also exciting are the possibilities that the concept of hidden space dimensions can be probed by collider as well as other experiments in not too distant future.

In this note, we point out another application of the extra dimensions. We show that the use of boundary conditions on fields in the extra dimensions provides a new way to break the parity symmetry of the left-right symmetric models. This idea is analogous to that of supersymmetry breaking by choosing different boundary conditions of various members of the supermultiplets advocated long ago by Scherk and Schwarz [9].

\footnotetext{
*e-mail:rmohapat@physics.umd.edu

†e-mail:aplorenz@Glue.umd.edu
} 
We show that in the case when the bulk field used is fermionic, starting with a completely parity symmetric Lagrangian in the brane with positive mass terms for the Higgs fields, the contributions of the bulk fermion fields leads to a radiative symmetry breaking of parity symmetry. In this framework, the parity breaking scale $v_{R}$ and the string scale, $M_{s t r}$ are of the same order if we use the string scale as the cutoff in divergent Feynman loops in the effective four dimensional field theory. For the sake of comparison we point out that in the literature there exist two ways to break left-right symmetry in the context of $S U(2)_{L} \times$ $S U(2)_{R} \times U(1)_{B-L}$ models: one is to use different masses for the Higgs fields $\chi_{L}, \chi_{R}$ thereby breaking parity softly [10] and a second one is to keep the weak Lagrangian exactly parity symmetric but looking for a parity asymmetric minimum which can exist for a domain of the parameters in the Higgs potential [11]. In both these cases, the value of the parity breaking scale is an arbitrary parameter. There is however a difference between these two methods in that the second case could apriori lead to the domain wall problem in the early universe, to cure which one may need additional physics inputs [12]. From the way the new mechanism suggested in this article is implemented, it appears that the domain wall problem should be absent in the present case.

We then discuss the process of neutrinoless double beta decay in the presence of extra dimensions and show that the higher Kaluza-Klein modes of the righthanded W-boson provide new contributions to this process. We compute these contributions and obtain correlated limits on $m_{W_{R}}$ and the inverse size of the extra dimensions. We find that due to already existing limits on $R^{-1}$ from the considerations of the standard model phenomena [7], the limits on $W_{R}$ remain same as in the case without the extra dimensions. Once the limits on the double beta lifetime improve, one could expect more stringent limits on $W_{R}$ as well as $R^{-1}$. This discussion applies regardless of how parity is broken.

\section{A TOY MODEL}

Let us start our discussion by considering a toy model with a discrete symmetry. Consider a model with a $Z_{2}$ symmetry under which two scalar fields $\chi_{L}$ and $\chi_{R}$ living in the $3+1$ dimensional brane go into each other. Let us assume that the brane is embedded in a $4+1$ dimensional spacetime. Consider a pair of bulk fields $\sigma_{L} \oplus \sigma_{R}$ which also go into each other under the $Z_{2}$ symmetry. The action for this system can be written as a sum of three terms:

$$
\mathcal{S}=\mathcal{S}_{4}\left(\chi_{L}, \chi_{R}\right)+\mathcal{S}_{5}\left(\sigma_{L}, \sigma_{R}\right)+\mathcal{S}_{45}\left(\chi_{L}, \chi_{R}, \sigma_{L}, \sigma_{R}\right)
$$

The detailed form of the four dimensional part of the action $\mathcal{S}_{4}$ is obvious; below we explicitly give the other terms:

$$
\begin{aligned}
\mathcal{S}_{5} & =\int d^{4} x d y\left[\partial^{M} \sigma_{L}^{\dagger} \partial_{M} \sigma_{L}+L \leftrightarrow R+\ldots\right] \\
\mathcal{S}_{45} & =\int d^{4} x\left[\chi_{L}^{\dagger} \chi_{L} \sigma_{L}(y=0)+L \leftrightarrow R\right]
\end{aligned}
$$

Now comes the crucial point of our paper. In order to evaluate the $\mathcal{S}_{45}$, we need to know the boundary conditions on the bulk fields $\sigma_{L, R}$. Suppose we impose the boundary conditions as follows: 


$$
\begin{array}{r}
\sigma_{L}(x,-y)=-\sigma_{L}(x, y) \\
\sigma_{R}(x,-y)=\sigma_{R}(x, y)
\end{array}
$$

The fields can then be Fourier expanded in the interval $-\pi R \leq y \leq+\pi R$ as follows:

$$
\begin{array}{r}
\sigma_{L}(x, y)=\frac{1}{\sqrt{\pi R}} \sum_{n} \sigma_{L}^{(n)} \sin \frac{n y}{R} \\
\sigma_{R}(x, y)=\frac{1}{\sqrt{2 \pi R}} \sigma_{R}^{(0)}+\frac{1}{\sqrt{\pi R}} \sum_{n \neq 0} \sigma_{R}^{(n)} \cos \frac{n y}{R}
\end{array}
$$

Now we see that if the brane on which the $\chi$ fields live is located in the bulk at the point $y=0$, then $\sigma_{L}(y=0)=0$. As a result the $3+1$ dimensional brane Lagrangian is not $Z_{2}$ symmetric anymore. When we compute the radiative corrections (cutting off the infinite integrals at the string scale), we find $Z_{2}$ asymmetric contributions to the physical parameters. In particular the self energy corrections to the $\chi$ mass terms will be asymmetric with only the $\chi_{R}$ receiving contributions of magnitude

$$
m_{1-\text { loop }_{R}}^{2} \sim \sum_{K K} \frac{M_{s t r}^{2}}{M_{s t r} R} \approx M_{s t r}^{2} .
$$

where we have used the fact that the number of KK modes contributing to the integral is roughly $M_{s t r} R$. Important point to note is that the bulk field flows in the loop and if we had a situation where the bulk field was a fermion field, this contribution to mass would be negative triggering break down of the discrete symmetry even if the tree level terms in the brane were positive. In that case the string scale and the scale of $Z_{2}$ symmetry breaking would necessarily be of same order. We will give an example of this type in the context of a realistic left-right symmetric model where the discrete symmetry on the fields in the bulk arise quite naturally.

\section{PARITY BREAKING IN A LEFT-RIGHT SYMMETRIC MODEL}

These models which have been extensively discussed in the literature are based on the gauge group $S U(2)_{L} \times S U(2)_{R} \times U(1)_{B-L}$ with quark and lepton assignments into left-right symmetric $\mathrm{SU}(2)$ doublets. We will not display this part of the Lagrangian explicitly but rather focus on the part that is relevant to our discussion of the symmetry breaking. We consider the nonsupersymmetric version of the model and use the left-right Higgs doublets $\chi_{L}(2,1+1) \oplus \chi_{R}(1,2,+1)$ to break the gauge symmetry. We will also include a bidoublet $\phi(2,2,0)$ to give mass to the charged fermions. We further assume that there is at least one bulk neutrino (denoted by $\nu_{B}$ ) which couples to the brane fields. Also for simplicity we will consider a five dimensional theory although this is not essential for the discussion of symmetry breaking.

Let us now discuss the parity transformation of the Higgs fields and the bulk field. We use the straightforward definition of parity under which $\psi_{L} \rightarrow \psi_{R}$ where $\psi$ denotes a typical fermion field (both in the bulk and the brane). The Higgs fields transform as $\chi_{L} \leftrightarrow \chi_{R}$.

As in the case of the toy model, the action will have three parts and we will use the same notation as in the case of the toy model. In the $3+1$ dimensional brane Lagrangian, in 
addition to the usual gauge invariant pieces, we will assume that the parity invariant mass term for $\chi_{L, R}$ fields is small ( $\sim m_{W}$ or smaller).

Let us write down the relevant terms in the action:

$$
\begin{aligned}
\mathcal{S}_{45} & =\int d^{4} x\left[\kappa \bar{L} \chi_{L} \nu_{B R}(x, y=0)+\kappa \bar{R} \chi_{R} \nu_{B L}(x, y=0)+\text { h.c. }\right] \\
\mathcal{S}_{5} & =\int d^{4} x d y \bar{\nu}_{B} \Gamma^{5} \partial_{5} \nu_{B}
\end{aligned}
$$

where $L^{T}=\left(\nu_{e L}, e_{L}\right)$ and $R^{T}=\left(\nu_{e R}, e_{R}\right)$

In order to break the $S U(2)_{R}$ gauge group, we need to give $\left\langle\chi_{R}^{0}\right\rangle=v_{R}$. In previous discussions of this [1], one searches for a domain of the coupling parameters in the Higgs potential

$$
\begin{aligned}
V\left(\chi_{L}, \chi_{R}\right)=\mu^{2}\left(\chi_{L}^{\dagger} \chi_{L}+\chi_{R}^{\dagger} \chi_{R}\right) & +\lambda_{+}\left(\chi_{L}^{\dagger} \chi_{L}+\chi_{R}^{\dagger} \chi_{R}\right)^{2} \\
& +\lambda_{-}\left(\chi_{L}^{\dagger} \chi_{L}-\chi_{R}^{\dagger} \chi_{R}\right)^{2}
\end{aligned}
$$

One finds that for $\lambda_{-}<0$, the minimum of the above potential is parity violating. What we will show is that using the new way we propose, one can break parity symmetry even for $\lambda_{-} \geq 0$.

Crucial to implementing our mechanism of parity breaking are the boundary conditions on the bulk field. We impose the following condition:

$$
\nu_{B}(x,-y)=-\gamma_{5} \nu_{B}(x, y)
$$

This implies that

$$
\begin{aligned}
& \nu_{B L}(x,-y)=+\nu_{B L}(x,+y) \\
& \nu_{B R}(x,-y)=-\nu_{B R}(x,+y)
\end{aligned}
$$

This allows us to write the following Fourier expansions for the $\nu_{B L, R}$ fields in the extra dimension $y$ which is compactified and assumed to be in the domain $-\pi R \leq y \leq \pi R$ as in the previous case.

$$
\begin{aligned}
& \nu_{B R}(x, y)=\frac{1}{\sqrt{\pi R}} \sum_{n} \nu_{B R}^{(n)} \sin \frac{n y}{R} \\
& \nu_{B L}(x, y)=\frac{1}{\sqrt{2 \pi R}} \nu_{B L}^{(0)}+\frac{1}{\sqrt{\pi R}} \sum_{n \neq 0} \nu_{B L}^{(n)} \cos \frac{n y}{R}
\end{aligned}
$$

Now we see that since the brane is located at the point $y=0, \nu_{B R}(x, y=0)=0$ and therefore the $L_{L} \chi_{L} \nu_{B}$ coupling vanishes. This induces the breaking of parity symmetry in the brane Lagrangian.

One practical consequence of this explicit breaking (induced by the bulk) is that the one loop self energy contribution from the $\nu_{B}$ intermediate state to the $\chi_{L}$ field vanishes whereas for the $\chi_{R}$, we get

$$
m_{\chi_{R}}^{2}(1-\text { loop }) \sim-\frac{1}{M_{s t r} R} \sum_{n} \int \frac{d^{4} k}{(2 \pi)^{4}} \frac{\left(k^{2}-p . k\right)}{k^{2}\left(k^{2}-2 p . k+p^{2}-\frac{n^{2}}{R^{2}}\right)}
$$


This integral has a quadratic dependence on the cut off which is independent of the mass of the leptons running into the loop. Therefore, all the KK modes contribute roughly with the same amount to the mass term. We estimate this to be $m_{\chi_{R}}^{2} \sim-a M_{s t r}^{2}$. Note that this contribution to the scalar mass is negative which therefore can trigger the breaking of parity symmetry leading to the relation

$$
v_{R} \sim M_{s t r}
$$

Thus this way of parity breaking enables us to relate the otherwise free parameter $v_{R}$ to more fundamental scales in the theory. Note that this new negative contribution to the self mass of the $\chi_{R}$ is present only in the presence of the bulk neutrino. For instance, in its absence the only self energy contribution to $\chi_{R}$ would come from the scalar self couplings and would be positive.

Admittedly the discussions given above have been very simpleminded with no pretense to rigor; however we believe that we have pointed out a fundamentally new way to discuss breaking of discrete symmetries. In fact it would be quite interesting to search for a model of $\mathrm{CP}$ violation that uses this mechanism.

Let us close this section with some brief comments on some other implications of our work.

(i) If we consider a five dimensional theory (or a theory with one large dimension and other dimensions with size $\sim M_{s t r}^{-1}$ ), then the relation between the Planck mass and the string scale becomes:

$$
M_{P \ell}^{2} \simeq M_{s t r}^{3} R
$$

where $\mathrm{R}$ is the size of the extra dimension. Since gravity experiments require that $R \leq 1$ $\mathrm{mm}$, this implies that $M_{s t r} \geq 10^{8} \mathrm{GeV}$. This would then imply that there is a lower limit on the right handed scale of $10^{8} \mathrm{GeV}$ in our way of breaking right handed symmetry. Here we have assumed that other extra dimensions have sizes of order $M_{s t r}^{-1}$ or larger; otherwise the lower limit on the string scale and hence the right handed scale becomes weaker. It is important to note that existence of millimeter size extra dimensions are compatible with the new way of parity breaking.

(ii) If we want to implement this way of parity breaking in models where the righthanded symmetry is broken by $B-L=2$ triplets $\left[13 \Delta_{R}(1,3+2) \oplus \Delta_{L}(3,1,+2)\right.$, then we have to put these multiplets in the bulk. This requires for consistency that $S U(2)_{L} \times S U(2)_{R} \times U(1)_{B-L}$ must also be in the bulk. This automatically implies that the bulk size cannot be larger than a $\mathrm{TeV}^{-1}[7]$. In this case, processes such as neutrinoless double beta decay receive new contributions and we discuss it in the next section.

(iii) This model leads to the profile of neutrino masses discussed in the Ref. [14]. Otherwise there would be an additional term that will mix the $\nu_{L}$ 's with the bulk neutrinos and that coupling has to be tuned down to an appropriate level to make the scheme of Ref. [14] work. (iv) In this theory the universe never reaches a symmetric parity invariant phase. As a result, there is no domain wall problem as already noted. There may be additional cosmological consequences of the bulk neutrinos, specially one might wonder about the impact of the heavy modes of the bulk neutrino. This depends on the picture of inflation in such models and at this stage of the development of the field, the answers are not clear. 
(v) The bulk neutrinos couple to the standard model matter only via the right handed Higgs fields, which being longitudinal modes of the $W_{R}$ are very heavy. Therefore, it is not possible to get any constraints on the bulk neutrino properties from known low energy electroweak data.

Finally, we note that the gauge couplings also receive one loop parity asymmetric corrections which therefore make the gauge couplings unequal. Their difference is connected to logarithm of the string scale and is therefore small (of the order of the usual RGE corrections).

\section{LEFT-RIGHT SYMMETRY IN HIGHER DIMENSIONS AND NEUTRINOLESS DOUBLE BETA DECAY}

In this section we discuss the case when the left-right gauge fields are in the bulk as are the Higgs fields that break the righthanded gauge symmetry. The fermions are in the brane and the usual seesaw mechanism $\left(\Delta_{R}(1,3+2)\right.$ breaking the righthanded symmetry) is used to get small neutrino masses. Parity could be broken by the usual way using the potential or by using the boundary conditions on Higgs fields propagating in the bulk. In this case, there will be higher Kaluza-Klein modes of the $W_{R}$ that will contribute to all processes where the right handed $\mathrm{W}$ used to contribute. In particular, it will contribute to neutrinoless double beta decay [15] via the exchange of $W_{R}$ and the heavy righthanded Majorana neutrino. For simplicity we will consider the case where masses of the $W_{R}$ and the righthanded neutrino are same i.e. $M_{W_{R}}=M_{N_{R}}$. In the presence of the KK models, the contribution to the strength of the neutrinoless double beta amplitude can be written as:

$$
M_{\beta \beta} \simeq \frac{G_{F}^{2}}{2} \frac{M_{W_{L}}^{4}}{M_{W_{R}}^{5}} f\left(M_{W_{R}}, R\right)
$$

where $R$ is the size of the extra dimension (we assume only one extra dimension to be important). We get

$$
\begin{aligned}
f\left(M_{W_{R}}, R\right) & =\left[1+2 \sum_{n} \frac{M_{W_{R}}^{2} R^{2}}{M_{W_{R}}^{2} R^{2}+n^{2}}\right]^{2} \\
& =\left[\pi M_{W_{R}} R \operatorname{coth}\left(\pi M_{W_{R}} R\right)\right]^{2}
\end{aligned}
$$

The present experimental bounds from the ${ }^{76}$ Ge experiment [16] on $f$ can be obtained using crude estimates of the nuclear matrix elements by $p_{F e r m i}^{3}$ and the result is

$$
\frac{M_{W_{L}}^{4}}{M_{W_{R}}^{5}} f\left(M_{W_{R}}, R\right) \leq 2 \times 10^{-7} \mathrm{GeV}^{-1}
$$

In Fig. 1 we plot the resulting correlated limits on $R^{-1}$ and $M_{W_{R}}$ for the present limit on the double beta amplitude from ${ }^{76} \mathrm{Ge}$ and an anticipated improvement on it by one order of magnitude in future. The regions to the right of the lines is allowed yielding lower limits on $R^{-1}$ and $M_{W_{R}}$.

One can see the asymptotic effects on the limit as follows: (i) $M_{W_{R}} R \ll 1$ : In this case, 


$$
f\left(M_{W_{R}}, R\right) \approx\left(1+\frac{\pi^{2} M_{W_{R}}^{2} R^{2}}{3}\right)^{2}
$$

Note that this is consistent with decoupling theorem in the limit of vanishing radius of the extra dimensions. We see that the KK modes do not have any effect on the limit on $M_{W_{R}}$ without them. This is the large $R^{-1}$ domain in the figure. In the opposite extreme, we have (ii) $M_{W_{R}} R \gg 1$ : In this case,

$$
f\left(M_{W_{R}}, R\right) \approx\left(\pi M_{W_{R}} R\right)^{2}
$$

For this case to hold, we must have $M_{W_{R}} \gg R^{-1}$; but already electroweak physics implies that $R^{-1} \gg 1-5 \mathrm{TeV}$ [7]. In this case also there are no stronger limits since as soon as $M_{W_{R}} \gg \mathrm{TeV}$, the coefficient of $f$ in Eq. (16) becomes very small and the double beta limit is always satisfied. The general quality of the constraints for arbitrary $M_{W_{R}}$ and $R$ are given in Fig. 1 from where it is clear that due to already existing constraints for the $S U(2)_{L}$ case, the constraints on $M_{W_{R}}$ are not very useful at this stage. If however the limit on the double beta amplitude goes down by another order of magnitude, we can start seeing more useful constraints(see the line to the far right in Fig. 1). There are now several experimental proposals such as GENIUS [17], CUORE [18] and MOON [19] which have the potential to give such limits. Further details on this question are now under study.

In conclusion, we have presented a new way to break parity (and other discrete symmetries) using extra dimensions [20,21]. We construct explicit examples of left-right symmetric models where this way of parity breaking is realized. We then discuss the effect of extra dimensions on neutrinoless double beta decay and find a correlated bound involving both the size of an extra dimension and the the $W_{R}$ scale.

Acknowledgements. The work of RNM is supported by a grant from the National Science Foundation under grant number PHY-9802551. The work of APL is supported in part by CONACyT (México). 


\section{REFERENCES}

[1] I. Antoniadis, Phys. Lett. B246 (1990) 377; I. Antoniadis, K. Benakli and M. Quirós, Phys. Lett. B331 (1994) 313.

[2] E. Witten, Nucl. Phys. B 471 (1996) 135; P. Horava and E. Witten, Nucl. Phys. B475 (1996) 94.

[3] J. Lykken, Phys. Rev. D 54 (1996) 3693.

[4] N. Arkani-Hamed, S. Dimopoulos and G. Dvali, Phys. Lett. B429 (1998) 263; Phys. Rev. D 59 (1999) 086004; I. Antoniadis, S. Dimopoulos, G. Dvali, Nucl. Phys. B516 (1998) 70; N. Arkani-Hamed, S. Dimopoulos and J. March-Russell, hep-th/9809124.

[5] K.R. Dienes, E. Dudas and T. Gherghetta, Phys. Lett. B436 (1998) 55; Nucl. Phys. B537 (1999) 47.

[6] K. Benakli, Phys. Rev. D 60 (1999) 104002,Phys. Lett. B447 (1999) 51; M. L. Graesser, hep-ph/9902310; T. Banks, A. Nelson and M. Dine, JHEP 9906 (1999) 014; D. Ghilencea and G.G. Ross, Phys. Lett. B442 (1998) 165; Z. Kakushadze, Nucl. Phys. B548 (1999) 205; C.D. Carone, Phys. Lett. B454 (1999) 70; A. Delgado and M. Quirós, hep-ph/9903400. P. H. Frampton and A. Răsin, Phys. Lett. B460 (1999) 313; G. Giudice, R. Rattazzi and J. Wells, Nucl. Phys. B544 (1999) 3; E. Mirabelli, M. Perelstein and M. Peskin, Phys. Rev. Lett. 82 (1999) 2236; T. Han, J. Lykken and R. J. Zhang, Phys. Rev. D 59 (1999) 105006; J. L. Hewett, Phys. Rev. Lett. 82 (1999) 47656; P. Mathews, S. Raychaudhuri and K. Sridhar, Phys. Lett. B450 (1999) 343; T. G. Rizzo, Phys. Rev. D 59 (1999) 115010; K. Aghase and N. G. Deshpande, Phys. Lett. B456 (1999) 60; K. Cheung and W. Y. Keung, hep-ph/9903294; T. Taylor and G. Veneziano, Phys. Lett. B212 (1988) 147; C. Burgess, L. Ibañez and F. Quevedo, Phys. Lett. B447 (1999) 257; A. Pérez-Lorenzana and R. N. Mohapatra, hep-ph/9904504; K. Huitu and T. Kobayashi, hep-ph/9906431; D. Dumitru and S. Nandi, hep-ph/9906514; C. Balazs, H. -J He, W. W. Repko, C. P. Yuan and D. A. Dicus, Phys. Rev. Lett. 83 (1999) 2112; T. Han, D. Rainwater and D. Zepenfield, hep-ph/9905423; A. Delgardo and M. Quiros, hep-ph/9903400; H.C. Cheng, B. Dobrescu and C. Hill, hep-ph/9906327; T. Rizzo and J. Wells, hep-ph/9906234; C. Carone, hep-ph/9907362; J. Lykken and S. Nandi, hep-ph/9908505; Nima Arkani-Hamed, Savas Dimopoulos, John March-Russell, hep-th/9908146.

[7] See for example P.Nath and M. Yamaguchi, hep-ph/9903298; hep-ph/9902323; M. Masip and A. Pomarol, Phys. Rev. D 60 (1999) 096005; W. Marciano, Phys. Rev. D 60 (1999) 093006.

[8] K. Benakli and S. Davidson, Phys. Rev. D 60 (1999) 025004; D. Lyth, Phys. Lett. B448 (1999) 191; P. C. Argyres, S. Dimopoulos and J. March-Russell, Phys. Lett. B 441 (1998) 96; N. Arkani-Hamed, N. Kaloper, S. Dimopoulos and J. March-Russell, hep-ph/9903239; hep-ph/9903224.

[9] J. Scherk and J. Schwarz, Nucl. Phys. B153 (1979) 61.

[10] R. N. Mohapatra and J. C. Pati, Phys. Rev. D11 (1975) 566, 2558.

[11] G. Senjanović and R. N. Mohapatra, Phys. Rev. D 12 (1975) 1502.

[12] See for instance R. N. Mohapatra and G. Senjanović, Phys. Rev. Lett. 42 (1979) 1651; B. Rai and G. Senjanović, Phys. Rev. D49 (1994) 2729.

[13] R. N. Mohapatra and G. Senjanović, Phys. Rev. Lett. 44 (1980) 912. 
[14] R. N. Mohapatra, S. Nandi and A. Pérez-Lorenzana, hep-ph/9907520; Phys. Lett B (to appear).

[15] R. N. Mohapatra, Phys. Rev. D 34 (1986) 909.

[16] L. Baudis et al. Phys. Rev. Lett. 83 (1999) 41.

[17] H. Klapdor-Kleingrothaus, hep-ex/9907040.

[18] O. Cremonesi, Nucl. Phys. B (Proc. Suppl.) 77 (1999) 369.

[19] H. Ejiri, Talk at the INT workshop on Neutrinos, Seattle (1999).

[20] For earlier work that uses spacetime topology to break parity, see J. Anandan, Phys. Rev. Lett. 81, 1363 (1998).

[21] Different ways to break parity using extra dimensions, see R. Erdem, Mod. Phys. Lett. A 13 (1998) 465. 


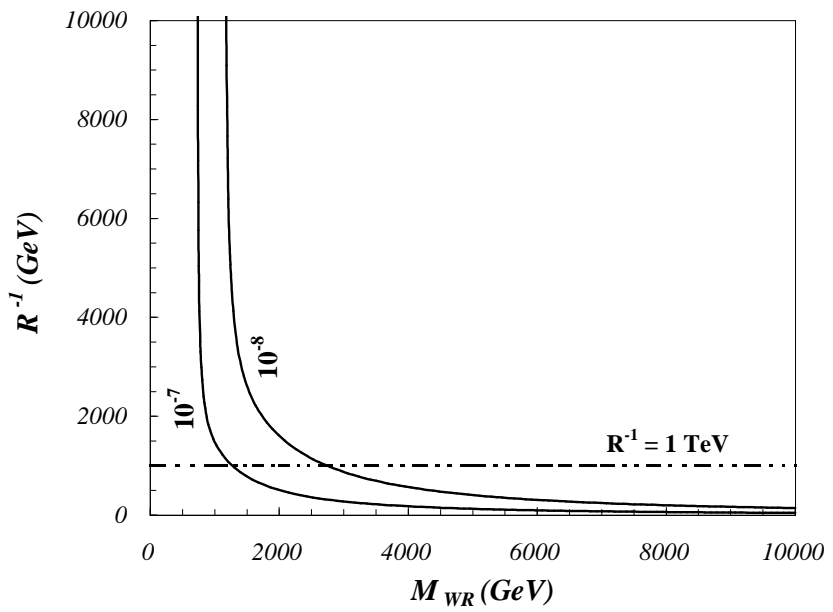

FIG. 1. Correlated limits for $M_{W_{R}}$ and $R$ from double beta decay and electroweak physics. The labels indicate the bounds for the amplitude (solid lines): $M_{W_{L}}^{4} f\left(M_{W_{R}}, R\right) / 2 M_{W_{R}}^{5}$ at the current limit $\left(10^{-7} \mathrm{GeV}^{-1}\right)$ and one order of magnitude lower; and for $R^{-1}=1 \mathrm{TeV}$ (dashed line). The allowed region is above both limits. 